\title{
Perilipin 5 is a novel target of nuclear receptor LRH-1 to regulate hepatic triglycerides metabolism
}

\author{
Rubee Pantha ${ }^{1}$, Jae-Ho Lee ${ }^{1}$, Jae-Hoon Bae ${ }^{1}$, Eun Hee Koh ${ }^{2}$, Minsang Shin ${ }^{3}$, Dae-Kyu Song ${ }^{1}$ E Seung-Soon Im $^{1, *}$ \\ ${ }^{1}$ Department of Physiology, Keimyung University School of Medicine, Daegu 42601, ${ }^{2}$ Department of Internal Medicine, Asan Institute for \\ Life Science, University of Ulsan College of Medicine, Seoul 05505, ${ }^{3}$ Department of Microbiology, School of Medicine, Kyungpook National \\ University, Daegu 41944, Korea
}

Liver receptor homolog-1 (LRH-1) has emerged as a regulator of hepatic glucose, bile acid, and mitochondrial metabolism. However, the functional mechanism underlying the effect of LRH-1 on lipid mobilization has not been addressed. This study investigated the regulatory function of LRH-1 in lipid metabolism in maintaining a normal liver physiological state during fasting. The Lrh-1 ${ }^{\mathrm{t} / \mathrm{f}}$ and LRH-1 liver-specific knockout (Lrh-1 ${ }^{\text {IKO }}$ ) mice were either fed or fasted for $24 \mathrm{~h}$, and the liver and serum were isolated. The livers were used for qPCR, westem blot, and histological analysis. Primary hepatocytes were isolated for immunocytochemistry assessments of lipids. During fasting, the Lrh-1 ${ }^{\mathrm{LKO}}$ mice showed increased accumulation of triglycerides in the liver compared to that in Lrh-1 ${ }^{\text {t/f }}$ mice. Interestingly, in the Lrh-1 ${ }^{\mathrm{LKO}}$ liver, decreases in perilipin 5 (PLIN5) expression and genes involved in $\beta$-oxidation were observed. In addition, the LRH-1 agonist dialauroylphosphatidylcholine also enhanced PLIN5 expression in human cultured HepG2 cells. To identify new target genes of LRH-1, these findings directed us to analyze the Plin5 promoter sequence, which revealed $-1620 /-1614$ to be a putative binding site for LRH-1. This was confirmed by promoter activity and chromatin immunoprecipitation assays. Additionally, fasted Lrh-1 ${ }^{\text {f/f }}$ primary hepatocytes showed increased co-localization of PLIN5 in lipid droplets (LDs) compared to that in fasted Lrh-1 ${ }^{\text {LKO }}$ primary hepatocytes. Overall, these findings suggest that PLIN5 might be a novel target of LRH-1 to mobilize LDs, protect the liver from lipid overload, and manage the cellular needs during fasting. [BMB Reports 2021; 54(9): 476-481]

*Corresponding author. Tel: +82-53-258-7423; Fax: +82-53-258-7412; E-mail: ssim73@kmu.ac.kr

https://doi.org/10.5483/BMBRep.2021.54.9.051

Received 20 April 2021, Revised 21 May 2021, Accepted 9 August 2021

Keywords: Fasting, Lipid droplet, Liver receptor homolog-1, Perilipin 5, Triglycerides

\section{INTRODUCTION}

Liver receptor homolog-1 ( $\mathrm{LRH}-1 / \mathrm{NR} 5 \mathrm{~A} 2)$ is a representative of the nuclear receptor $5 \mathrm{~A}$ subfamily of orphan nuclear receptors, mainly expressed in the liver, pancreas, ovary, and intestine (1). It is the principal regulator of glucose, bile acid, and cholesterol metabolism with varied biological roles extending from regulation of the cell cycle to the maintenance of steroid homeostasis $(2,3)$. In the pancreas, LRH-1 with pancreas transcription factor stimulates the expression of genes encoding pancreatic digestive enzymes and secretory proteins (4). Moreover, $\mathrm{LRH}-1$ regulates the maturation of ovarian follicles and ovulation in the ovary (5) and is responsible for mitochondrial function by regulating cytochrome p450, family 11 , subfamily a, polypeptide 1 and cytochrome p450, family 11 , subfamily b, polypeptide 1 in the intestinal epithelium (6). In the liver, LRH-1 is involved in mitochondrial biogenesis and $\beta$-oxidation through the regulation of peroxisome proliferator-activated receptor gamma coactivator 1-alpha gene expression (PGC-1 $\alpha$ ) (7). It is also involved in maintaining the pool of arachidonoyl phospholipids, which are important for normal lipid homeostasis in the liver (8). In addition, LRH-1 liver-specific knockout (Lrh- ${ }^{\mathrm{LKO}}$ ) mice show endoplasmic reticulum stress-induced fatty liver, indicating that LRH-1 plays a major role in triglyceride (TG) accumulation in the liver (9).

Excess TGs in the liver are mainly reserved within lipid droplets (LDs) (10). LDs are used to balance lipid storage and utilization and are strongly regulated in a cell type-specific manner (11). As LDs modulate low intracellular free fatty acid levels, they play a crucial role in protecting the liver from lipotoxicity caused by excess fatty acids in nutritional stress conditions (10, 12). In addition, the mobilization of LDs reflects the metabolic state of cells and also indicates changes in the LD-related proteins that contribute to the regulation of lipid metabolism and lipid homeostasis (13).

The perilipin (PLIN) protein family, which attaches to LDs, is a representative group of LD-associated proteins that utilizes the stored lipids of LDs via lipolysis $(10,11)$. It is composed of five members, named PLIN1-5 and is classified based on stability in a free state. PLIN1 and PLIN2 rapidly degrade in a free state; however, they exist when bound to LDs. The remaining

ISSN: 1976-670X (electronic edition)

Copyright (c) 2021 by the The Korean Society for Biochemistry and Molecular Biology

(c) This is an open-access article distributed under the terms of the Creative Commons Attribution Non-Commercial License (http://creativecommons.org/licenses/by-nc/4.0) which permits unrestricted non-commercial use, distribution, and reproduction in any medium, provided the original work is properly cited. 
proteins from the family, PLIN3, PLIN4, and PLIN5, are found either free in the cytosol or lining the LDs (14). PLIN1 is found in white and brown adipose tissue (WAT and BAT), whereas PLIN2 and PLIN3 are distributed in many cell types, and PLIN2 is especially observed in hepatocytes. PLIN4 is expressed in cardiomyocytes, adipocytes, and myocytes, and PLIN5 is usually confined within tissues or cells with high oxidative capacity, namely the liver, heart, BAT, and muscle $(11,14,15)$.

Among the PLIN family, PLIN5 has emerged as indispensable for adjusting lipid abundance. It is highly active upon fatty acid treatment in cultured cells, with a high-fat diet, and upon prolonged fasting (10). Prolonged fasting is also known to increase TGs accumulation in the liver $(16,17)$ due to increased adipose tissue lipolysis (18). Therefore, PLIN5 is also regarded as the regulator of TGs metabolism (19). The overexpression of PLIN5 in cells enhances the expression of genes encoding proteins involved in aerobic catabolism and promotes both TGs storage and fatty acid oxidation. Thus, PLIN5 rapidly mobilizes energy by sensing the nutrient demand (20).

The nuclear receptor $\mathrm{LRH}-1$ is activated during nutritional stress and is involved in protecting the liver from lipid overload by increasing the $\beta$-oxidation. Even though $\mathrm{LRH}-1$ regulates mitochondrial biogenesis and lipid metabolism, its regulatory mechanism and function under fasting conditions has not been completely addressed. Therefore, in this study, the regulatory function of LRH-1 was investigated during fasting state by utilizing Lrh- ${ }^{\mathrm{fff}}$ and Lrh- ${ }^{\mathrm{LKO}}$ mice to verify the function of $\mathrm{LRH}-1$ in cellular energy demands and lipid overloading state.

\section{RESULTS}

\section{LRH-1 mitigates hepatic lipid overload by inducing the} expression of PLIN5 and fatty acid oxidation-related genes To understand the function of LRH-1 in regulating TGs during fasting, Lrh- $1^{\mathrm{f} / \mathrm{f}}$ and Lrh- ${ }^{\mathrm{LKO}}$ mice were either fed or starved for $24 \mathrm{~h}$, and the liver (Fig. 1A) and serum were isolated to examine hepatic and serum TGs levels. Initially, serum $\beta$-hydroxybutyrate levels was measured in both Lrh- $1^{\text {t/f }}$ and Lrh- $1^{\text {LKO }}$ mice to confirm the fasting condition. As expected, the fasting condition has increased serum $\beta$-hydroxybutyrate levels but did not show significant differences between the genotypes (Supplementary Fig. 2A). The livers from Lrh- $1^{\mathrm{LKO}}$ mice starved for $24 \mathrm{~h}$ displayed higher accumulation of lipids compared to that in livers of starved Lrh- ${ }^{\mathrm{f} / \mathrm{f}}$ mice in Oil red-O staining (Fig. $1 \mathrm{~B}, \mathrm{C})$, implying the necessity for further analysis of lipid levels in the liver and serum. Interestingly, fasted Lrh-1 ${ }^{\text {LKO }}$ mice exhibited remarkably escalated hepatic TGs levels compared to those in fasted Lrh- $1^{\mathrm{f} / \mathrm{f}}$ mice. However, there was no significant difference between the fed mice of either genotype (Fig. 1D). In contrast, hepatic cholesterol was not altered between the genotypes in either fed or starved conditions (Fig. 1E). Surprisingly, Lrh- $1^{\mathrm{LKO}}$ mice either fed or starved showed a notable decrease in serum TGs levels compared to those in Lrh-1 ${ }^{\mathrm{f} / \mathrm{f}}$ mice (Fig. 1F). However, serum cholesterol and non-

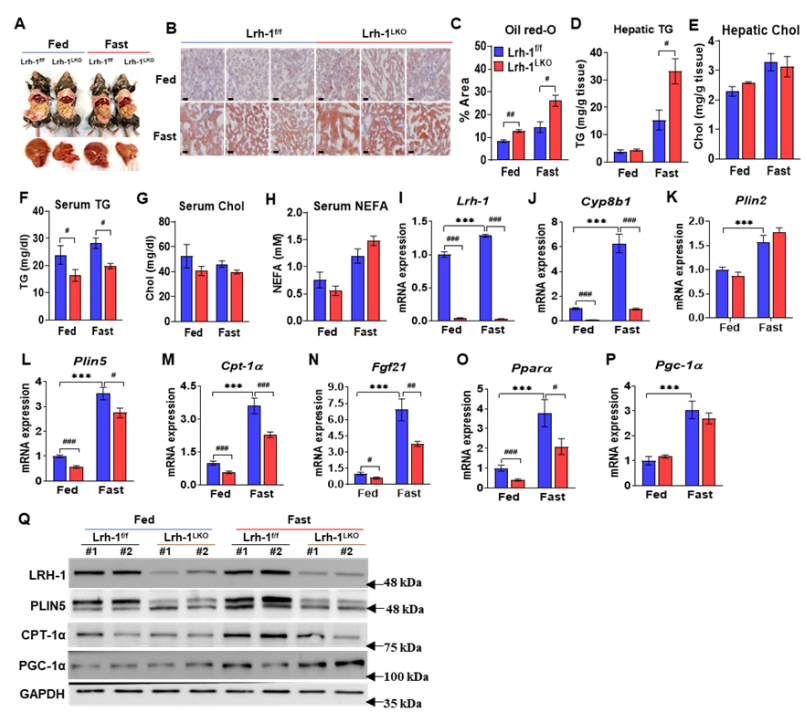

Fig. 1. Increased hepatic lipid contents in fasted $\mathrm{Lrh}-1^{\mathrm{LKO}}$ mice via decreased $\beta$-oxidation genes. All experiments were performed in 24 h-fasted or fed Lrh- $1^{\mathrm{f} / \mathrm{h}}$ and Lrh- $1^{\text {LKO }}$ mice. (A) Dissection image of $24 \mathrm{~h}$-fasted or fed Lrh-1 ${ }^{\mathrm{f} / \mathrm{f}}$ and Lrh- ${ }^{\mathrm{LKO}}$ mice along with their isolated livers. (B) Oil red-O staining of liver tissue (scale bar $=30 \mu \mathrm{m}$ ). (C) Quantification of staining in \% area shown in B. (D) Hepatic triglycerides levels. (E) Hepatic cholesterol levels. (F) Serum triglycerides levels. (G) Serum cholesterol levels. (H) Serum NEFA levels $(n=4 /$ group). (I, J) mRNA expression level of $L r h-1$ and its target gene. $(\mathrm{K}, \mathrm{L})$ mRNA expression levels of Plin2 and Plin5 respectively. $(\mathrm{M}, \mathrm{N})$ mRNA expression of Cpt-1 $\alpha$ and Fgf 21 respectively. $(\mathrm{O}, \mathrm{P})$ mRNA expression of Ppar $\alpha$ and Pgc- $1 \alpha$, respectively ( $n=5 /$ group). (Q) Western blot for protein analysis. GAPDH was used as a loading control. " $\mathrm{P}<0.05,{ }^{\# \#} \mathrm{P}<0.01$, \# $\mathrm{P}<0.001$, Lrh-1 ${ }^{\text {f/f }}$ vs. Lrh-1 ${ }^{\text {LKO }}$, $* * * \mathrm{P}<0.001$, Lrh- ${ }^{\mathrm{f} / \mathrm{f}}$ fed vs. Lrh-1 ${ }^{\mathrm{t} / \mathrm{f}}$ fast.

esterified fatty acids (NEFA) levels were not altered significantly (Fig. 1G, H). These findings suggest that the loss of LRH-1 results in a buildup of lipids in the liver, indicating that $\mathrm{LRH}-1$ might be a key regulator in balancing the hepatic lipid content.

To discover new potent target genes of LRH-1 involved in lipid metabolism, the mRNA and protein expression of various genes was examined in liver samples. The expression levels of Lrh-1 (Fig. 1I) and its target gene (Fig. 1J) were highly reduced in Lrh- ${ }^{\text {LKO }}$ livers which confirmed Lrh- ${ }^{\text {LKO }}$ mice.

In the liver, PLIN2 and PLIN5 are highly expressed (21) therefore, mRNA expression was measured. However, Plin2 did not display significant difference between the genotypes in either fed or fasted conditions (Fig. 1K). In the liver, PLIN5 is known to regulate lipid metabolism (10) by promoting or inhibiting the hydrolysis of LDs (22). Next, the mRNA and protein expression of PLIN5 was measured. Interestingly, as Lrh-1 expression was augmented, the Plin5 level also increased in the livers of Lrh- $1^{\mathrm{f} / \mathrm{f}}$ fasted mice. However, the expression of Plin5 was diminished markedly in the livers of either fed or starved Lrh- $1^{\mathrm{LKO}}$ mice (Fig. 1L).

Additionally, TGs and $\beta$-oxidation are strongly interconnected 
with lipid metabolism. To understand the different liver phenotypes in Lrh- $1^{\text {f/f }}$ and Lrh- $1^{\mathrm{LKO}}$ mice, the expression of genes involved in fatty acid $\beta$-oxidation was measured. Carnitine palmitoyltransferase-1 alpha (Cpt-1 $\alpha)$, a regulator of fatty acid $\beta$-oxidation (23) and fibroblast growth factor 21 (Fgf21), an oxidation enhancer and lipogenesis inhibitor (24) were expressed at remarkably lower levels in the livers of either fed or starved Lrh-1 ${ }^{\text {LKO }}$ mice. Nevertheless, expression was highly escalated in livers of Lrh- $1^{\mathrm{t} / \mathrm{f}}$ fasted mice (Fig. $1 \mathrm{M}, \mathrm{N}$ ). Similarly, peroxisome proliferator-activated receptor alpha (Pparo), a transcription factor that regulates Cpt-1 $\alpha$ and Fgf21, significantly increased gene expression levels in Lrh-1/f/f fast mice. However, gene expression levels were significantly decreased in both fed and fasted Lrh- ${ }^{\mathrm{LKO}}$ mice (Fig. 1O). Gene expression levels of PgC-1 $\alpha$ was increased during fasting but did not show significant differences between the genotypes (Fig. 1P). In addition, dialauroylphosphatidylcholine (DLPC), an LRH-1 agonist increased fatty acid oxidation-related genes in PLIN5 knockdown human cultured HepG2 cells (Supplementary Fig. 4). Moreover, protein levels of LRH-1, PLIN5, CPT- $1 \alpha$, and PGC- $1 \alpha$ were measured in the liver samples (Fig. 1Q). Protein levels of LRH-1 and PLIN5 were remarkably decreased in either fed or fasted Lrh- $1^{\text {LKO }}$ mice. Also, protein levels of CPT- $1 \alpha$ decreased in Lrh- $1^{\text {LKO }}$ mice. These findings suggest that PLIN5 is a putative target of $\mathrm{LRH}-1$, and that the loss of $\mathrm{LRH}-1$ reduces the expression of $\beta$-oxidation-related genes.

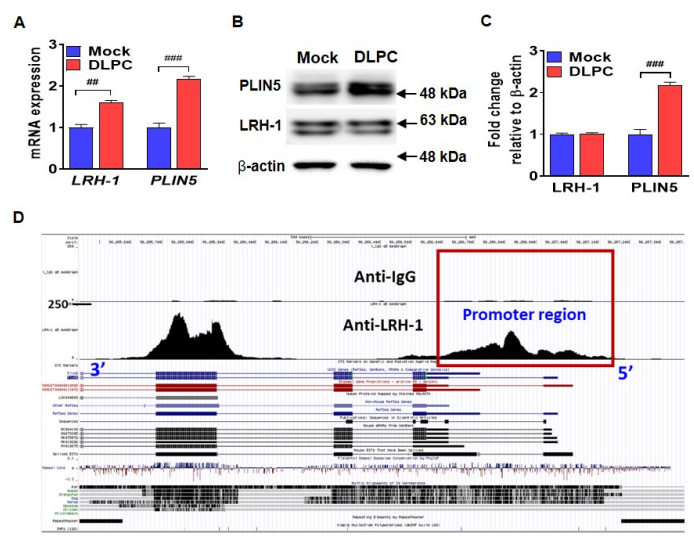

Fig. 2. LRH-1 agonist elevates PLIN5 gene expression. (A) mRNA expression of $L R H-1$ and PLIN5 gene after DLPC treatment. (B) Western blot for protein analysis of PLIN5 and LRH-1 after DLPC treatment. (C) LRH-1 and PLIN5 protein fold change relative to $\beta$-actin which was used as a loading control. These experiments were performed in triplicate. ${ }^{\# \#} \mathrm{P}<0.01,{ }^{\# \# \# P}<0.001$, Mock vs. DLPC. (D) LRH-1 response elements on the mouse liver chromosome were obtained from the LRH-1. ChIP-Seq data and identified peaks that mapped to the mouse Plin5 promoter using the UCSC Genome Browser. ChIP-Seq, chromatin immunoprecipitation sequencing.

\section{LRH-1 agonist amplifies PLIN5 gene expression due to putative LRH-1 responsive elements (LRE) in PLIN5 promoter region}

To determine whether PLIN5 is regulated by an LRH-1 agonist, human cultured HepG2 cells were treated with $100 \mu \mathrm{M}$ DLPC for $24 \mathrm{~h}$ and the gene expression and protein levels of PLIN5 were measured. In the presence of DLPC, PLIN5 mRNA expression (Fig. 2A) and protein levels (Fig. 2B, C) increased significantly. Although, DLPC treatment did not alter LRH-1 protein levels (Fig. 2C), the mRNA expression was increased (Fig. 2A). These observations indicate that PLIN5 could be regulated by an exogenous agonist of $\mathrm{LRH}-1$, implying that $\mathrm{LRH}-1$ guides PLIN5.

To discover potent LRH-1-regulated genes, putative LRE in the Plin5 promoter sequence were analyzed by Chromatin immunoprecipitation sequencing (ChIP-Seq) analysis using a published mouse ChIP-Seq data set for LRH-1 (25). As expected, the ChIP-Seq analysis of Plin5 resulted in the identification of LRE peaks in the promoter region (Fig. 2D). Furthermore, Plin5 proximal promoter sequences were mapped utilizing the UCSC

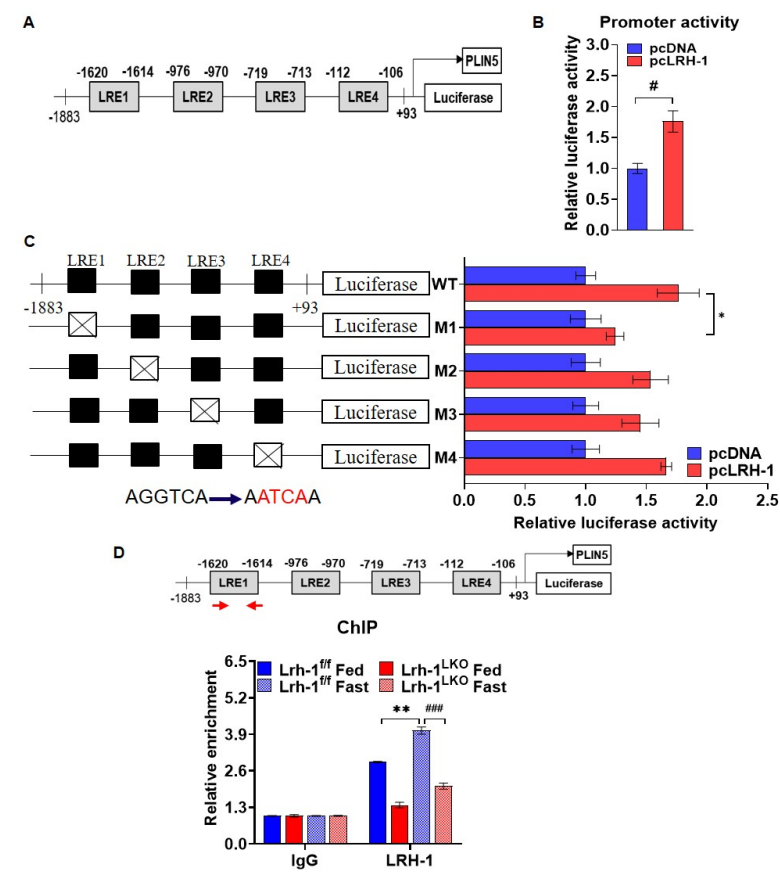

Fig. 3. LRE in the Plin 5 promoter is responsive to LRH-1. (A) Representation of putative LRE in Plin5 promoter region. (B) HEK-293T cells were transfected with pmPLIN5 containing the Plin5 promoter upstream of the luciferase reporter gene along with a pcDNA or $\mathrm{LRH}-1$ expression vector. (C) $\mathrm{LRH}-1$ deletion mutants from the Plin5 promoter region co-transfected with pcDNA or $\mathrm{LRH}-1$ expression vector. The deleted sequences were shown in boxes. These experiments were performed in triplicate. ${ }^{\#} \mathrm{P}<0.05$, pcDNA vs pcLRH-1, $* \mathrm{P}<0.05$ WT pcLRH-1 vs M1 pcLRH-1. (D) ChIP assay measured in $24 \mathrm{~h}$-fasted or fed Lrh-1 ${ }^{\text {t/f }}$ and Lrh-1 ${ }^{\text {LKO }}$ livers. ( $\mathrm{n}=3 /$ group). ${ }^{\# \#} \mathrm{P}<0.001$, Lrh- ${ }^{\mathrm{f} / \mathrm{f}}$ fast vs. Lrh-1 $1^{\mathrm{LKO}}$ fast, $* * \mathrm{P}<0.01$, Lrh- $1^{\mathrm{t} / \mathrm{f}}$ fed vs. Lrh-1 $1^{\mathrm{t} / \mathrm{f}}$ fast. 
genome browser to identify the putative LRE. The Plin5 promoter region was found to have four putative LRE with direct orientations $(-112 /-106,-719 /-713,-976 /-970$, and $-1620 /-1614$ from the transcription starting site; Supplementary Fig. 1A).

\section{LRH-1 stimulates PLIN5 promoter activity}

To confirm whether LRH-1 controls Plin5 at a transcriptional level by binding its promoter, the Plin5 promoter region was cloned upstream of the luciferase expression reporter gene (Fig. 3A). The Plin5 promoter construct was co-transfected with or without the LRH-1 expression plasmid and cells were treated with $100 \mu \mathrm{M}$ DLPC. The Plin5 promoter activity increased significantly in the presence of the LRH-1 expression vector and DLPC (Fig. 3B). In addition, to distinguish the main LRE among the four putative sites in the Plin5 promoter, each putative LRE was deleted from the construct. The deletion at the putative site -1620 / -1614 diminished luciferase activity in response to LRH-1 in comparison to that with the other sites (Fig. 3C).

This finding shows that $-1620 /-1614$ in the Plin5 promoter region was the conserved site for $\mathrm{LRH}-1$ binding and its removal in the construct diminished the response to LRH-1. Furthermore, binding of the LRH-1 at the $-1620 /-1614$ site was verified by a ChIP assay performed on liver samples from $24 \mathrm{~h}$-fasted and fed Lrh-1 $1^{\mathrm{f} / \mathrm{f}}$ and Lrh- ${ }^{\mathrm{LKO}}$ mice. When the sample was treated with the LRH-1 antibody, enrichment of the LRE -1620 / - 1614 was markedly elevated in livers of fasted Lrh- $1^{\mathrm{f} / \mathrm{f}}$ mice compared to that in fed Lrh-1/f/ mice. Moreover, there were significant differences between the genotypes for either fed or starved mice (Fig. 3D). In addition, electrophoretic mobility shift assay (EMSA) was also performed to confirm the binding of $\mathrm{LRH}-1$ at the $-1620 /-1614$ region of the Plin5 promoter sequence. The radiolabeled DNA probe corresponding to $-1620 /-1614$ region which includes LRE were incubated with LRH-1 overexpressed nuclear extract proteins. Thus, DNA-LRH-1 complex resulted in supershift while unlabeled cold DNA reduced the supershift (Supplementary Fig. 3). Moreover, Lrh- $1^{\mathrm{LKO}}$ mice decreased the enrichment of LRH-1 on Plin5 gene exon region (Supplementary Fig. 1B). These results suggested that the $-1620 /-1614$ site in the Plin5 promoter region is responsible for the transcriptional regulation by $\mathrm{LRH}-1$.

\section{LRH-1 controls PLIN5 to regulate LDs in mouse hepatocytes during nutritional stress}

To evaluate the regulation of LDs via $\mathrm{LRH}-1$, primary hepatocytes were isolated from Lrh-1 $1^{\mathrm{ft/}}$ and Lrh- ${ }^{\mathrm{LKO}}$ mice to perform BODIPY staining. Primary hepatocytes were grown either in complete (fed) or fasting media to understand the regulatory mechanism of LRH-1 during fasting in the liver. In the fasting media, PLIN5 surrounding the LDs was more abundant in the Lrh- $1^{\mathrm{f} / \mathrm{f}}$ hepatocytes than in the Lrh-1 ${ }^{\mathrm{LKO}}$ hepatocytes. Moreover, fasting condition increased LDs number in Lrh- $1^{\mathrm{LKO}}$ hepatocytes relative to that in Lrh- $1^{\mathrm{f} / \mathrm{f}}$ hepatocytes. Nevertheless, the sizes of the LDs in Lrh- ${ }^{\mathrm{f} / \mathrm{f}}$ hepatocytes were found to be distinct and increased compared to that in Lrh-1 ${ }^{\text {LKO }}$ hepatocytes (Fig. 4A, B). Addition- ally, in fasting condition, the co-localization of PLIN5 in LDs was increased in Lrh-1/f/ hepatocytes compared to that in complete media (Fig. 4C).

Next, to verify alterations in the lipid quantity in the liver and serum, the mRNA expression of microsomal triglyceride transfer protein (Mttp), a key gene responsible for the assembly and release of lipoproteins, was measured. The Lrh- ${ }^{\text {LKO }}$ mice either fed or starved showed remarkably decreased Mttp mRNA expression compared to that in Lrh- $1^{\mathrm{f} / \mathrm{f}}$ mice (Fig. 4D). Furthermore, expression of the apolipoprotein $\mathrm{B}(A p o B)$ gene, a principal component present in very low-density lipoproteins (VLDLs) was measured. However, there was no significant differences between the genotypes (Fig. 4D). In addition, PLIN5 dependent hepatic TGs accumulation during fasting in Lrh-1 ${ }^{\mathrm{LKO}}$ mice was confirmed by overexpressing PLIN5 in the Lrh- $1^{\text {LKO }}$ mice (Supplementary Fig. 2B). The PLIN5 overexpressed Lrh-1 ${ }^{\text {LKO }}$ mice significantly decreased hepatic TGs accumulation compared to that of the control Lrh- ${ }^{\text {LKO }}$ mice (Fig. 4E). Taken together, PLIN5 recovers the fasting-induced TGs accumulation in the Lrh- ${ }^{\text {LKO }}$ mice, and the loss of LRH-1 decreases PLIN5 co-localization in the LDs. Moreover, LRH-1 deficiency increases TGs in the liver by decreasing TGs secretion, leading to a surplus in the LDs.

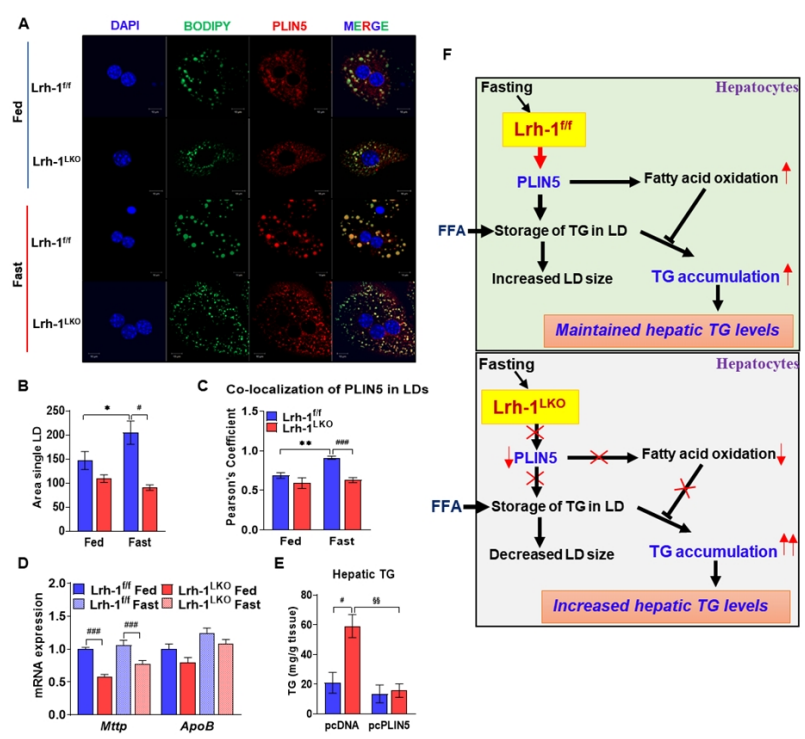

Fig. 4. LRH-1 mobilizes hepatic LDs via PLIN5. (A) BODIPY staining in primary hepatocytes grown in complete (fed) or fasting media followed by overnight incubation of PLIN5 antibody. (B) Quantification data for the size of LDs shown in A. (C) Co-localization of PLIN5 in LDs shown in $A$ ( $n=3 /$ treatment group). (D) mRNA expression of genes related to VLDL secretion measured in $24 \mathrm{~h}$-fasted or fed Lrh-1 ${ }^{\text {f/f }}$ and Lrh- $1^{\text {LKO }}$ livers. ( $n=5 /$ group). (E) Hepatic triglycerides levels by PLIN5 overexpression in Lrh- $1^{\text {/f/ }}$ and Lrh- $1^{\text {LKO }}$ mice ( $\mathrm{n}=4$ /group). \#\# $\mathrm{P}<0.001$, " $\mathrm{P}<0.05$, Lrh-1 ${ }^{\mathrm{t} / \mathrm{f}}$ vs Lrh-1 ${ }^{\mathrm{LKO}}, * * \mathrm{P}<0.01,{ }^{*} \mathrm{P}<0.05$, Lrh- $^{\mathrm{f} / \mathrm{f}}$ fed vs. Lrh- $1^{\mathrm{f} / \mathrm{f}}$ fast, ${ }^{\$ S} \mathrm{P}<0.01$, Lrh- $^{\text {LKO }}$ pcDNA vs Lrh- ${ }^{\text {LKO }}$ pcPLIN5. (F) Scheme illustrating the role of $\mathrm{LRH} 1$ in the liver during fasting. 


\section{DISCUSSION}

Hepatic LRH-1 is a transcriptional regulator of glucose metabolism and bile acid homeostasis (26). This study discovered PLIN5 as a direct target of $\mathrm{LRH}-1$ and explored the function of $\mathrm{LRH}-1$ in the liver during fasting. In this study, fasting increased the accumulation of liver TGs more readily in the Lrh- $1^{\text {LKO }}$ livers compared to that in Lrh-1 ${ }^{\text {f/f }}$ livers. The elevation of hepatic TGs in the liver might be due to a decrease in $\beta$-oxidation. As expected, livers of Lrh- $1^{\mathrm{LKO}}$ mice either fed or starved showed the markedly diminished expression of key genes responsible for $\beta$-oxidation, as well as their enhancer gene, indicating the possible accumulation of lipids in the liver. Recent studies on Lrh- ${ }^{\text {LKO }}$ mice revealed that LRH-1 promotes $\beta$-oxidation and mitochondrial biogenesis (7). Incidentally, this result coincides with the previous findings in PLIN5 $5^{\mathrm{LKO}}$ mice, demonstrating elevated hepatic TGs levels and a reduction in fatty acid oxidation in the liver (10). However, these findings were in contrast with the reported research performed by Wang et al. (27). They reported decreased TGs in the liver and increased $\beta$-oxidation in the whole body PLIN5-KO mice. Together, this suggests close phenotypic similarity between LRH-1 and PLIN5 due to the transcriptional regulation of PLIN5 by LRH-1.

Interestingly, starved Lrh- ${ }^{\mathrm{LKO}}$ mice exhibited a decrease in serum TGs levels compared to that in Lrh- ${ }^{\mathrm{f} / \mathrm{f}}$ mice. Altered liver and serum TGs levels between the genotypes were also observed, which might be due to a decrease in TGs secretion from the liver (28). Therefore, the genes involved in VLDL secretion from the liver were measured. The gene expression of Mttp, a key player in VLDL secretion was markedly decreased in the livers of Lrh- $1^{\mathrm{LKO}}$ mice; however, $A p o B$ was unaltered. Furthermore, a decrease in TGs secretion was reported in PLIN5 ${ }^{\mathrm{LKO}}$ mice (10). Collectively, these data suggest that MTTP might be responsible for decreasing serum TGs in the livers of Lrh- $1^{\mathrm{LKO}}$ mice.

Transcription factors often bind and sense lipid molecules (29). $\mathrm{LRH}-1$ binds to the $-1620 /-1614$ binding sequence in the Plin5 promoter region for its transcriptional regulation, which was confirmed by promoter activity, ChIP assays and EMSA. In addition, DLPC, as an agonist of LRH-1, which has a previously established role in the synthesis of bile acids and reduction of hepatic TGs (30), increases the mRNA expression of PLIN5. Therefore, these findings indicate that $\mathrm{LHH}-1$ regulates PLIN5 at the transcriptional level.

PLIN5 balances fatty acid requirements to meet cellular needs, protecting mitochondria during extreme fatty acid flux with low-energy demands and encouraging fatty acid mobilization and oxidation with high-energy demands (31). Based on BODIPY staining, starved Lrh-1/f/ hepatocytes demonstrated the utilization of LDs, whereas lipids were accumulated in the fasted Lrh- $1^{\text {LKO }}$ hepatocytes. Furthermore, PLIN5 and BODIPY staining clearly resulted in more intense red and green fluorescence, respectively, implying the co-localization of PLIN5 in LDs during starvation in Lrh-1 ${ }^{\mathrm{fff}}$ hepatocytes. This indicates that the loss of LRH-1 decreases PLIN5 co-localization in LDs and increases the lipid content. Surprisingly, in the starved Lrh- $1^{\mathrm{f} / \mathrm{f}}$ hepatocytes, the size of the LDs was increased and distinct compared to that in starved Lrh- $1^{\text {LKO }}$ hepatocytes. However, the quantity of lipids was increased in the fasted Lrh- $1^{\text {LKO }}$ hepatocytes. A previous study reported a decrease in the amount and size of LDs in the whole body PLIN5-KO mice (27). Nevertheless, in this study, the number of LDs increased in the Lrh- $1^{\mathrm{LKO}}$ mice. PLIN5 regulates both the storage and usage of TGs and is regarded as metabolically protective (32). In fasted Lrh- ${ }^{\mathrm{f} / \mathrm{f}}$ hepatocytes, $\mathrm{LRH}-1$ regulates PLIN5 to protect the liver by increasing the influx of TGs within the LDs. As a result, this increases the size of LDs. In addition, it promotes fatty acid oxidation to meet cellular energy demands during starvation, resulting in fewer LDs. In contrast, the lack of LRH-1 in the Lrh- ${ }^{\text {LKO }}$ mice attenuated PLIN5 expression, resulting in small-sized LDs and increasing their numbers (Fig. 4F). This might be the mechanism underlying the changes in the size and quantity of LDs, respectively. Furthermore, the overexpression of PLIN5 decreased the fasting-induced TGs accumulation in the Lrh-1 ${ }^{\text {LKO }}$ liver, which confirmed the significant role of PLIN5 expression in regulating TGs metabolism. Overall, these observations indicate that LRH-1 regulates PLIN5 to mobilize LDs and balances hepatic lipid contents.

In summary, this study uncovered the function of LRH-1 in the liver during fasting and presents a novel target of $\mathrm{LRH}-1$ in the liver. Results further suggest the necessity of LRH-1 in lipid management to protect the liver from lipid accumulation. In the liver, LRH-1 regulates PLIN5 to mobilize lipids and maintains this balance during fasting conditions. Additionally, $\mathrm{LRH}-1$ regulates PLIN5 to equilibrate the cellular needs and storage of lipids, thus protecting the liver from metabolic diseases associated with a fatty liver. Although $\mathrm{LRH}-1$ is involved in managing the lipid content in the liver, further studies are required to assess the possible targeting of this molecule for the treatment of nonalcoholic steatohepatitis. Thus, this study might be a platform to elucidate the mechanism underlying the treatment of nonalcoholic steatohepatitis and could be beneficial for the protection of the liver.

\section{MATERIALS AND METHODS}

The detailed methods are described in the "Supplementary Information".

\section{ACKNOWLEDGEMENTS}

We acknowledge Dr. Timothy F. Osborne at Johns Hopkins University School of Medicine for kindly providing Lrh- $1^{\mathrm{t} / \mathrm{f}}$ mice. This study was supported by grants of the Korea Research Foundation, an NRF grant funded by the Korea Government (MSIP) (2019R1A2C2085302, NRF-2021R1A4A1029238) and KMPC (2013M3A9D5072550). 


\section{CONFLICTS OF INTEREST}

The authors have no conflicting interests.

\section{REFERENCES}

1. Fayard E, Auwerx J and Schoonjans K (2004) LRH-1: an orphan nuclear receptor involved in development, metabolism and steroidogenesis. Trends Cell Biol 14, 250-260

2. Stein S, Lemos V, Xu P et al (2017) Impaired SUMOylation of nuclear receptor $\mathrm{LRH}-1$ promotes nonalcoholic fatty liver disease. J Clin Invest 127, 583-592

3. Schwaderer J, Phan TS, Glockner A et al (2020) Pharmacological LRH-1/Nr5a2 inhibition limits pro-inflammatory cytokine production in macrophages and associated experimental hepatitis. Cell Death Dis 11, 154

4. Holmstrom SR, Deering T, Swift GH et al (2011) LRH-1 and PTF1- $L$ coregulate an exocrine pancreas-specific transcriptional network for digestive function. Genes Dev 25, 1674-1679

5. Duggavathi R, Volle DH, Mataki $C$ et al (2008) Liver receptor homolog 1 is essential for ovulation. Genes Dev 22, 1871-1876

6. Mueller M, Cima I, Noti M et al (2006) The nuclear receptor LRH-1 critically regulates extra-adrenal glucocorticoid synthesis in the intestine. J Exp Med 203, 2057-2062

7. Choi S, Dong B, Lin CJ et al (2020) Methyl-sensing nuclear receptor liver receptor homolog-1 regulates mitochondrial function in mouse hepatocytes. Hepatology 71, 1055-1069

8. Miranda DA, Krause WC, Cazenave-Gassiot A et al (2018) $\mathrm{LRH}-1$ regulates hepatic lipid homeostasis and maintains arachidonoyl phospholipid pools critical for phospholipid diversity. JCl Insight 3, e96151

9. Mamrosh JL, Lee JM, Wagner $M$ et al (2014) Nuclear receptor $\mathrm{LRH}-1 / \mathrm{NR} 5 \mathrm{~A} 2$ is required and targetable for liver endoplasmic reticulum stress resolution. Elife 3, e01694

10. Keenan SN, Meex RC, Lo JCY et al (2019) Perilipin 5 deletion in hepatocytes remodels lipid metabolism and causes hepatic insulin resistance in mice. Diabetes 68, 543-555

11. Kimmel AR and Sztalryd C (2014) Perilipin 5, a lipid droplet protein adapted to mitochondrial energy utilization. Curr Opin Lipidol 25, 110-117

12. Khor VK, Shen WJ and Kraemer FB (2013) Lipid droplet metabolism. Curr Opin Clin Nutr Metab Care 16, 632-637

13. Pol A, Gross SP and Parton RG (2014) Review: biogenesis of the multifunctional lipid droplet: lipids, proteins, and sites. J Cell Biol 204, 635-646

14. Langhi C, Marquart TJ, Allen RM and Baldan A (2014) Perilipin- 5 is regulated by statins and controls triglyceride contents in the hepatocyte. J Hepatol 61, 358-365

15. Tan $Y$, Jin $Y$, Wang $Q$, Huang J, Wu X and Ren $Z$ (2019) Perilipin 5 protects against cellular oxidative stress by enhancing mitochondrial function in HepG2 cells. Cells 8, 1241

16. Yokota S, Nakamura K, Ando M et al (2014) Acetylcholinesterase (AChE) inhibition aggravates fasting-induced triglyceride accumulation in the mouse liver. FEBS Open
Bio 4, 905-914

17. Li Y, Chao X, Yang L et al (2018) Impaired fasting-induced adaptive lipid droplet biogenesis in liver-specific Atg5-deficient mouse liver is mediated by persistent nuclear factorlike 2 activation. Am J Pathol 188, 1833-1846

18. Geisler CE, Hepler C, Higgins MR and Renquist BJ (2016) Hepatic adaptations to maintain metabolic homeostasis in response to fasting and refeeding in mice. Nutr Metab (Lond) 13, 62

19. Asimakopoulou A, Engel KM, Gassler N et al (2020) Deletion of perilipin 5 protects against hepatic injury in nonalcoholic fatty liver disease via missing inflammasome activation. Cells 9, 1346

20. Gallardo-Montejano $\mathrm{VI}$, Saxena G, Kusminski $\mathrm{CM}$ et al (2016) Nuclear Perilipin 5 integrates lipid droplet lipolysis with PGC-1alpha/SIRT1-dependent transcriptional regulation of mitochondrial function. Nat Commun 7, 12723

21. Nocetti D, Espinosa A, Pino-De la Fuente F et al (2020) Lipid droplets are both highly oxidized and Plin2-covered in hepatocytes of diet-induced obese mice. Appl Physiol Nutr Metab 45, 1368-1376

22. Du J, Hou J, Feng J et al (2019) Plin5/p-Plin5 guards diabetic CMECs by regulating FFAs metabolism bidirectionally. Oxid Med Cell Longev 2019, 8690746

23. Weber M, Mera P, Casas J et al (2020) Liver CPT1A gene therapy reduces diet-induced hepatic steatosis in mice and highlights potential lipid biomarkers for human NAFLD. FASEB J 9, 11816-11837

24. Lin X, Liu YB and Hu H (2017) Metabolic role of fibroblast growth factor 21 in liver, adipose and nervous system tissues. Biomed Rep 6, 495-502

25. Chong HK, Biesinger J, Seo YK, Xie X and Osborne TF (2012) Genome-wide analysis of hepatic LRH-1 reveals a promoter binding preference and suggests a role in regulating genes of lipid metabolism in concert with FXR. BMC Genomics 13, 51

26. Oosterveer MH, Mataki C, Yamamoto $\mathrm{H}$ et al (2012) LRH-1dependent glucose sensing determines intermediary metabolism in liver. J Clin Invest 122, 2817-2826

27. Wang C, Zhao Y, Gao X et al (2015) Perilipin 5 improves hepatic lipotoxicity by inhibiting lipolysis. Hepatology 61, 870-882

28. Khalil A, Cevik SE, Hung S, Kolla S, Roy MA and Suvorov A (2018) Developmental exposure to 2,2',4,4'-tetrabromodiphenyl ether permanently alters blood-liver balance of lipids in male mice. Front Endocrinol (Lausanne) 9, 548

29. Seitz C, Huang J, Geiselhoringer AL et al (2019) The orphan nuclear receptor $\mathrm{LRH}-1 / \mathrm{NR} 5 \mathrm{a} 2$ critically regulates $\mathrm{T}$ cell functions. Sci Adv 5, eaav9732

30. Lee JM, Lee YK, Mamrosh JL et al (2011) A nuclear-receptor-dependent phosphatidylcholine pathway with antidiabetic effects. Nature 474, 506-510

31. Mason RR and Watt MJ (2015) Unraveling the roles of PLIN5: linking cell biology to physiology. Trends Endocrinol Metab 26, 144-152

32. Harris LA, Skinner JR, Shew TM, Pietka TA, Abumrad NA and Wolins NE (2015) Perilipin 5-driven lipid droplet accumulation in skeletal muscle stimulates the expression of fibroblast growth factor 21. Diabetes 64, 2757-2768 